\title{
Data Acquisition System Research Based on Mobile Agent in Network Management
}

\author{
Yan $\mathrm{Li}^{1, \mathrm{a}}$, Li Zhu ${ }^{2, \mathrm{~b}}$ \\ ${ }^{1}$ Jiangxi Science and Technology Normal University, Nanchang, China \\ ${ }^{2}$ Jiangxi Science and Technology Normal University, Nanchang, China \\ a*hanter3@163.com, b'zhuli_821@163.com
}

Keywords: Mobile agent; Data acquisition; Network management

\begin{abstract}
In traditional network management, data acquisition realized by polling and traps will cause huge waste of network bandwidth, and cause delay and congestion problems. To solve this problem, based on mobile agent platform aglets, this paper developed a data acquisition system, which realized transparent access of distributed configuration data acquisition. This design can reduce the computational load of network management, and reduce bandwidth demands by a large number of information exchanges.
\end{abstract}

\section{Introduction}

Data acquisition is the basis of reliable network management, which is a relatively independent module, mainly to provide the necessary data for network management and issue control commands to the managed object. The realization of network management function cannot leave management information acquisition and query. With the development of network, the complexity and heterogeneity increase, and necessary information transport for network management rapidly grows. How to construct a flexible, efficient underlying data acquisition system is very important. A good data acquisition system can separate upper management function system and managed objects, making the management system structure level more clearly. Traditional data acquisition is carried out based on SNMP polling and traps, and a huge amount of data is transmitted between network management stations and managed agents, which not only causes the waste of network bandwidth, but the resulting delay and congestion problem also interfere with the normal operation of network management. Although the network management protocol SNMP proposed method to handle large amounts of MIB information, such as Get Bulk, but it did not effectively solve the problem. So in this paper, a data acquisition system based on mobile agent is introduced. Mobile agent mobility and autonomy can well reduce the computational load of network management, and implement the transparent access of distributed configuration data acquisition.

\section{Mobile Agent}

\section{A. The concept of mobile agent}

Mobile agent is a kind of special agent, which can migrate independently and automatically in heterogeneous networks and distributed computing environment, carry information or search for appropriate information resources, in-place process information, and agent for the user to complete a variety of tasks such as information transmission, knowledge discovery, and information transform [1]. Mobile agent also supports heterogeneous computer software and hardware environment, asynchronous communication and calculation, and provides a unique architecture, distributed computation to complete a task. The user can create multiple agents, and run them on the same or different nodes at the same time, distribute a single node load to multiple nodes in a network, and make small system have the ability to deal with large-scale and complicated problems. Mobile agent has the ability of planning next steps according to target, network communication ability and server load and other factors dynamically. Intelligent routing can optimize network and computing 
resources, realize load balance, improve problem solving speed, and avoid blind access to resources [2].

\section{B. Mobile agent platform aglets}

At present, a lot of mobile agent platforms with different architecture and implementation method have developed [3], Aglet is one of the most successful and comprehensive platform, which provides a simple and comprehensive mobile agent programming model. It provides a dynamic and effective communication mechanism between agents, and it also provides a set of detailed and easy to use security mechanism. Aglet is produced in the form of threads on one machine, which can suspend at any time of the work, and then the whole aglet can be assigned to another machine, and then restart the mission [4]. Because aglet is thread, so it will not consume too many system resources.

Aglet provides some packages as follows [5]:

1) com.ibm.Aglet: this package defines aglet interface (AgletContext AgletProxy, MessageManager) and class (Aglet, AgletID AgletInfo, Aglettub, FutureReply, Message, ReplySet), provides common functions managing the sending and receiving of aglet context and agents information.

2) com.ibm.Aglet.event: including three Listener interfaces: Clone, Mobility, Persistency, in which Clone is for copying aglet, Mobility is for dispatch or recall aglet, Persistency is for suspension or wake aglet. This package achieves the life cycle of object model.

3) com.ibm.Aglet.system: mainly provide control Context interface ContextListener and management functions on aglet operation layer.

4) com.ibm.Aglets.util: contains some common classes such as AgletAudioClip, AgletImageData, ImageComponent and Utils, which provides effective management audio and image functions.

5) com.ibm.Aglets.patterns includes common design styles, for example, MasterSlave, Messenger - Receiver and Notifier - Notification etc. [6].

In addition, it also provides MASIF extending packages and classes.

\section{Data Acquisition System Based on Mobile Agent}

A. Data collection system framework

Distributed configuration information collection based on mobile aglet basically has the following several parts: server, client, internal network, and migration between each node performs information acquisition and processing of mobile agent. System framework is shown in Figure 1:

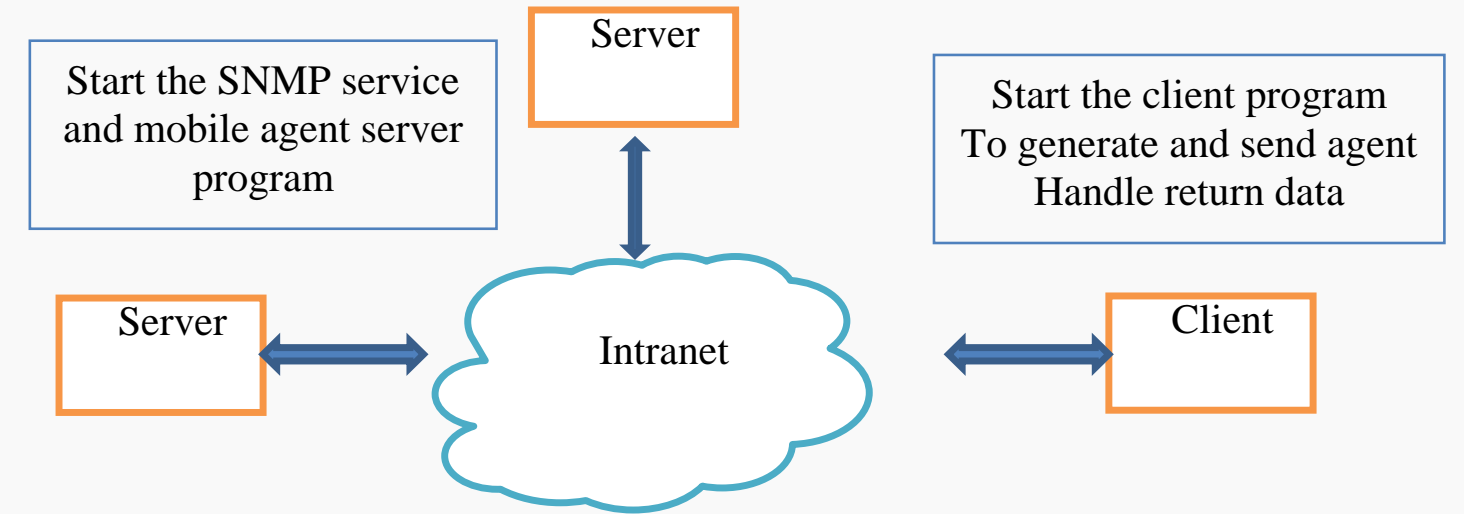

Figure 1 Data acquisition system framework based on mobile aglet

As shown in Figure 1, in distributed configuration information acquisition system based on mobile aglet, server is installed server program, and the program provides aglet of running environment and safety management to start the SNMP service on the server at the same time. All the aglet threads from the client to the server run in the server program environment. When the client collects configuration information, processing is through the query interface to submit queries, and the work flow of system configuration information collection is as follows:

1) By the user query interface, submit queries, including destination address list, data types, and mobile agent way of return. 
2) The client program has a static aglet (Data Collection Window Aglet) which is used to receive the user's query. The aglet in our developed system is responsible for generating user query interface, receiving the user query request, and is responsible for the information sent to mobile aglet of gathering configuration information (Data Collection Aglet class implementation), these information includes user entered target address, gathering information type and mobile agent way of return.

3) Static aglet (Data Collection Window Aglet) is responsible for generating and sending mobile aglet, handing over the acquisition plan to the aglet (Data Collection Aglet), receiving and processing Data returned by the mobile agent.

4) Mobile agent carries acquisition plan and migrates to the target server, obtains the corresponding configuration data information, and returns the data back to the client according to user specified way.

5) The results of data are showed on the client side. Die after mobile agent returns.

B. System implementation

a. The establishment of server environment

Each server needs to install server program, which provides aglet running background execution environment. Server program project files are stored in SNMP Manager Folder. When installing a server program, first aglet packages and JDK need to be installed on the server, second is to modify Client. bat, Server. bat batch files’ AGLET_HOME and JDK_HOME path, LOCALCLASSPATH resources path required is according to the specific conditions of the machine. The aglets version used in this experiment is V2.0, JDK is jdk2.5.0, which are installed on the machine D dish root directory. So in Client. bat and Server. bat files, there are the following two settings:

set AGLET_HOME=D:aglets202 , set JDK_HOME=D:Program FilesJavajdk1.5.0

b. Client program design

Configuration information acquisition system structure based on mobile agent

Generate and send

Configuration management
information center

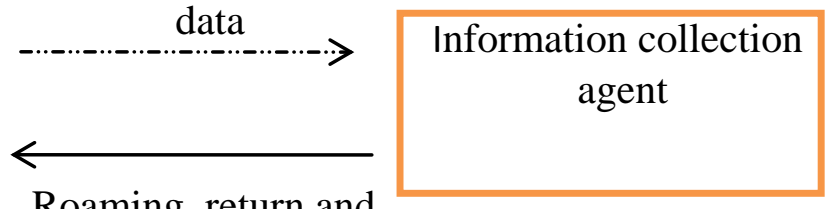

Roaming, return and summary data

Figure 2 System structure

As shown in Figure 2, the system is composed of "configuration management information center" and "information collection agents" two parts. User configuration information management center designates to gather configuration information of the host (IP address), forming (internal) acquisition host IP address list. Then, configuration management information center generates an information collection agent, and sends it out, the agents carried determined list of host addresses. Then, information collection agents move to every Host according to the Host address list carried in order, through mobile agent access facilities provided SNMP network management platform, basic configuration of the Host information (including RFC1213 group information of the system, Host-MIB Host devices information, storage information, install software). Agent can directly get the information (via aglets message) and transfer it back to configuration management information center, which can be temporarily stored in a carrying agent within back center (way designated by the user before sending). When the agent accessed all the hosts, and returned to center, agent releases automatically and terminates. Finally, the configuration information management center summarizes information collected, and displays to the user.

2. Configuration information management center design

Configuration management information center is a aglets client program, which completes the following functions: set acquisition target host list, content, data access method; generate and send information collection agency; collect information collection agency sent back to configuration 
information (aglets news), and the information can be sent back in agent process, or sent after the agent returns.

3. The design of configuration information collection agent

Information collection agent also has no aglets interface, which is composed of configuration information management center accesses each host according to order specified by the target host address list, and gathers configuration information.

When moving to a host, the basic working process is as follows:

1) Remove the machine IP address from the target host list;

2) Access to set collection content MIB values by SNMP facilities provided by mobile agent network management server;

3) If the set returns information directly, data will be collected into an aglets message, and send it back to the configuration management information center; otherwise, the information is spooled in the agent's memory;

4) Check if the target host list is empty. If not empty, move to the first host in the list of addresses; In turn, if a host is unreachable, set a time interval, if timeout, return information which indicates that the host is unreachable, and in turn to the next host to collect information. Finally, package together and return to management center. If empty, move back to the host where configuration information management center is.

5) When moving back to configuration information management center after the host, if set summary returns information, gather configuration information data into an aglets message, and send it to the configuration information management center. Finally, send a collection end configuration information management center, and then die.

\section{System Response Time Performance Analysis}

With comparison in response time performance while reading configuration data based on mobile agent and based on traditional SNMP method; use the developed system to test, then test the response time under the two structures.

When the managed equipment is concentrated in the same subnet, response time of mobile agent method is better than that of method based on SNMP. While reading the same amount of data, the increase of the number of hosts increases the response time gap gradually, so the response time of mobile agent features are superior to that of SNMP method.

Read the influence of the amount of data on response time, and analyze response time in network. It can be seen that when the amount of data read is little, method based on SNMP has certain advantages, but with the increase of amount of data, the method based on mobile agent shows advantages gradually. The greater the amount of data read, the more obvious advantages are. A straightforward explanation is that while reading amount of data is rare, the mobile agent spends more time on their own processing, such as source mobile agent system collection operation and coding, decoding of mobile agent on the target machine, instantiation, and activation time; when reading data volume is larger, mobile agent can migrate in high speed local area network (LAN), and the method of SNMP has to access multiple times, especially consume time on meter reading, so the processing time may be longer, and the advantages of mobile agent are more obvious.

\section{Conclusion}

This paper studies data acquisition system based on mobile agent in network management. The system is based on mobile agent platform aglets of IBM. Experimental environment includes two servers; the user realizes mobile agent access to configuration data distributed in two servers through the client. The success operation of the system shows that using mobile agent technology to access distributed configuration data is feasible and effective. At the same time, comparing with the performance of traditional method based on SNMP data collection, the experimental results show that when the number of hosts are more centralized or with greater amount of host managed, using mobile 
agent technology manifests the superiority on delay. In the same experiment environment, when collecting the same number of hosts, the greater the amount of data collected, the mobile agent delay is less than that of traditional method based on SNMP.

\section{References}

[1] Zhang Yunyong. Mobile agent technology [M]. Beijing: Tsinghua University Press, 2003.309-310.

[2] Shang Zonghai, Li Qi, Zhao Bao. Local area network (LAN) information acquisition and preprocessor based on mobile agent IBM [J]. Information Network Security. 2013 (3) : 64-67.

[3] Dong Hongbin, Shi Chunyi. Mobile agent technology research [J]. Journal of Computer Science. 2006 (4). 58-61.

[4] Cui Jianqun, Xiao Debao, Hao Mobing. Mobile agent applications and implementation in performance management data acquisition [J]. Computer Engineering. 2003 (9) : 72-74.

[5] Zou Qiming, Yu Tao. Manufacturing grid resources reliability data acquisition based on mobile agent [J]. Computer Engineering. 2006 (11) : 119-120.

[6] Wang Xi, Zhang Yuming. Data acquisition problems research and design based on mobile agent in campus network management [J]. Computer and Modern. 2004 (5) : 70-72. 